\section{Streptobacillus moniliformis mitral valve endocarditis and septic arthritis: the challenges of diagnosing rat-bite fever endocarditis}

\author{
Daisy Torres-Miranda, ${ }^{1}$ \\ Mahdi Moshgriz, ${ }^{2}$ Marc Siegel $^{1}$ \\ ${ }^{1}$ Department of Medicine, George \\ Washington University, Medical Faculty \\ Associates, Washington, DC; \\ ${ }^{2}$ Department of Pathology, George \\ Washington University Medical Center, \\ Washington, DC, USA
}

\begin{abstract}
Streptobacillus moniliformis, the cause of rat-bite fever (RBF) in the United States, has rarely been reported as a cause of infectious endocarditis. In the majority of previously reported cases, the diagnosis was clinically-based in patients with underlying valvular abnormalities in the setting of positive blood culture for Streptobacillus moniliformis. We report a case of native valve endocarditis secondary to Streptobacillus moniliformis in a woman with a mitral valve vegetation but negative blood cultures where the diagnosis was established using molecular diagnostics on the valvular tissue.
\end{abstract}

\section{Case Report}

A previously healthy 52 year-old African American female presented to the hospital with confusion. According to her partner, she had been complaining of constant pain in the right knee and ankle for three days, associated with generalized weakness. There was no history of trauma to the extremity. She had no known allergies and was not taking any medications. She had no history of recreational drugs, no recent travel and was only sexually active with one male partner.

On presentation, the patient was lethargic and oriented to self-only. Her temperature on admission was $39.4^{\circ} \mathrm{C}$, blood pressure $92 / 54 \mathrm{mmHg}$, heart rate 96 beats per minute and respiratory rate 18 breathes per minute, with an oxygen saturation of $96 \%$ on ambient air. On examination, the right knee and right ankle demonstrated swelling, warmth, and erythema. A punctate opening in the heel of right foot with no surrounding erythema was noted. Laboratory studies revealed leukocytosis (white blood cell count 22,600 cells $/ \mathrm{mL}, 88 \%$ neutrophils, $6 \%$ bands), hyponatremia (sodium 125 $\mathrm{mEq} / \mathrm{L}$ ), and hypokalemia (potassium 2.8 $\mathrm{mEq} / \mathrm{L}$ ). Inflammatory markers were elevated (erythrocyte sedimentation rate 85 $\mathrm{mm} / \mathrm{hr}$, C-reactive protein $>270 \mathrm{mg} / \mathrm{dL}$ ). Radiography of the right knee and ankle showed osteoarthritic changes with mild soft tissue swelling but no evidence of any acute pathology. Doppler ultrasound was negative for deep vein thrombosis. Arthrocentesis of the right knee yielded purulent synovial fluid (white blood cell count of 73,110 cells $/ \mathrm{mL}, 87 \%$ neutrophils). Gram stain showed no organisms. No arthrocentesis was performed on the right ankle.

Intravenous vancomycin and piperacillin-tazobactam were initiated for presumed septic arthritis and the following day the patient was taken to the operating room for washout of the right knee. This again showed purulent synovial fluid (white blood cell count 63,440 cells/mL, $94 \%$ neutrophils) and the Gram stain again revealed no organisms. The synovial fluid culture revealed growth of Gram-negative bacilli that could not be speciated using standard laboratory techniques. We used trypticase soy agar (TSA) with 5\% sheep blood, chocolate agar, Columbia CNA agar, MacConkey agar, and thioglycollate agar with vitamin $\mathrm{K}$ and Hemin. S. moniliformis grew best on chocolate agar after 4 days in anaerobic condition. No standardized methods have been established to determine $S$. moniliformis antimicrobial susceptibility, and since $S$. moniliformis there has been no documented resistance to penicillin, susceptibility testing was not performed.

The patient became afebrile on the third hospital day and the intravenous antibiotics were switched to ceftriaxone alone. However the patient had persistent leukocytosis, with a white blood cell count range from 14,000 to 16,000 cells/mL. A new cardiac murmur was subsequently auscultated and a transesophageal echocardiogram revealed a $1.4 \times 1.3 \mathrm{~cm}$ mass on the atrial side of the mitral valve with perforation and severe mitral regurgitation (Figure 1). All blood cultures remained no growth after 5 days incubation. Serologies for Bartonella henselae, Bartonella quintana and Brucella were negative. Polymerase chain reaction (PCR) for Coxiella burnetii was negative.

Due to difficulty in speciating the Gram-negative bacillus that grew from the synovial culture, Matrix-Assisted Laser Desorption Ionization Time-Of-Flight Mass Spectrometry (MALDI-TOF MS) was performed on one of the colonies, which identified the organism as Streptobacillus
Correspondence: Daisy Torres-Miranda, 2150

Pennsylvania Ave, NW, Suite 8-436, Washington, DC 20037, USA.

Tel.: +1.202.741.2234

E-mail: datorres@gwu.edu

Key words: Endocarditis; rat bite fever; Streptobacillus moniliformis.

Acknowledgments: we acknowledge that all authors have contributed to this work and have seen and approved the manuscript.

Conflicts of interest: the authors declare no conflicts of interest.

Received for publication: 23 April 2018.

Revision received: 19 June 2018.

Accepted for publication: 27 June 2018.

This work is licensed under a Creative Commons Attribution-NonCommercial 4.0 International License (CC BY-NC 4.0).

(C) Copyright D. Torres-Miranda et al., 2018 Licensee PAGEPress, Italy

Infectious Disease Reports 2018; 10:7731 doi:10.4081/idr.2018.7731

moniliformis. The patient was taken for mitral valve repair and the mitral valve leaflet showed rare clumps of bacteria on Brown-Hopps stain. However, there was again no growth on culture. The valve tissue was sent to the Centers for Disease Control and Prevention where 16S rRNA sequencing (Microseq, Applied Biosystems, Foster City, CA) was performed and demonstrated molecular evidence of Streptobacillus moniliformis

On further questioning, the patient and her partner admitted to living in an apartment with a rodent infestation but had no known contact with any rodents. The patient was treated with ceftriaxone $2 \mathrm{~g}$ intravenously daily for 6 weeks post valve repair. She was discharged after 5 weeks of hospitalization and made a full recovery.

\section{Discussion}

Rat-bite fever (RBF) is caused by $S$. moniliformis in North America, while Spirillum minus is the predominant cause in Asia. S. moniliformis is a fastidious, facultative anaerobic, pleomorphic, filamentous, Gram-negative rod bacteria found commensally in the throat and nasopharynx of rodents. ${ }^{1-6}$ Human infection can result from a bite or scratch from an infected rat or by consumption of contaminated food or water. ${ }^{7,8}$ Consistent with our patient, approximately $30 \%$ of the patients recall no 
bite or other exposure. ${ }^{4,6}$ Studies suggest that $S$. moniliformis may have a predilection for synovial and serosal surfaces. ${ }^{4,9}$

Symptoms of RBF occur three days to three weeks after infection. Symptoms include malaise, vomiting, fever, a morbilliform rash and polyarthralgias. ${ }^{1-7}$ The prevalence of RBF endocarditis is unclear but is considered to be rare. Including this case, there have been only 26 cases of $S$. moniliformis native valve endocarditis reported in the medical literature. In a review of 16 cases of RBF endocarditis, $50 \%$ of the patients had preexisting valvular damage, most commonly due to rheumatic heart disease. ${ }^{10}$ Embolic phenomena are rarely reported. ${ }^{6}$

The overall mortality rate of untreated RBF ranges from $7 \%$ to $13 \% .^{2,4-5}$ However RBF endocarditis is associated with significantly higher mortality rates, as high as $50 \%$ in some reviews. ${ }^{4,10}$ We estimated a mortality rate of $43 \%$ when we reviewed all the reported cases where patient outcomes were reported. The majority of these deaths occurred in cases that had received either non-targeted antibiotic therapy or inadequate antibiotic dosing. ${ }^{10-12}$

There have been three reviews of all of the previously reported cases of $S$. moniliformis endocarditis from 1915 to 2014 . $^{6-7,10}$ Since these reviews, two more cases of RBF infectious endocarditis have been reported. ${ }^{13,14}$ The diagnosis of endocarditis was most often established based on the clinical presentation, that included fever, rashes, and joint pains, a cardiac murmur, often without demonstrating a vegetation on echocardiogram, and a positive blood culture for S. moniliformis. ${ }^{6-7}$

In the majority of previously reported cases, $S$. moniliformis has grown in routine blood culture bottles. However, it has been reported that sodium polyanethol sulfonate

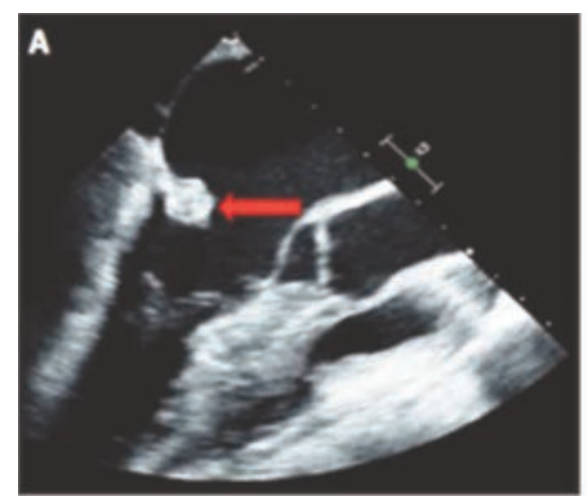

Figure 1. A) Transesophageal echocardiogram image showing a $1.4 \times 1.3 \mathrm{~cm}$ mass on the atrial side of the posterior leaflet of the mitral valve (solid arrow). B) Real time 3-dimensional echocardiogram image showing the mitral vegetation (solid arrow) and the $4 \mathrm{~mm}$ perforation of the posterior mitral valve leaflet (arrowhead).

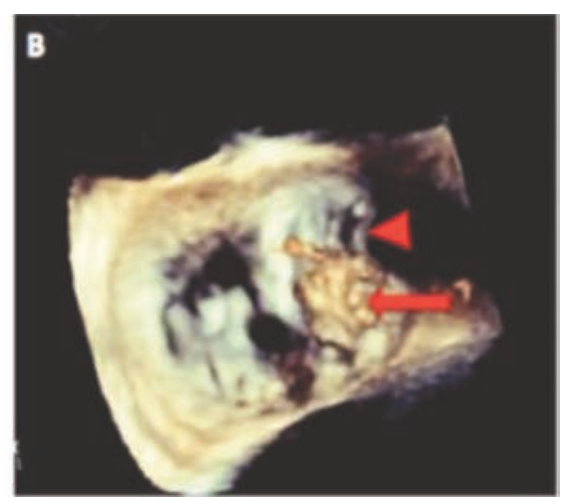

(SPS), the anticoagulant that is in most commercial aerobic blood culture bottles, can inhibit the growth of $S$. moniliformis. ${ }^{2,4-}$

5 Nevertheless, S. moniliformis has been successfully recovered from SPS containing blood culture bottles. ${ }^{6,15}$ Agar-based culture of $S$. moniliformis requires incubation with $5-10 \%$ carbon dioxide and TSA enriched media, while no serologic tests are currently available, making diagnosis challenging using standard practices. . $^{3,4-5}$

Because of challenges in microbiological diagnosis of RBF, molecular diagnostic techniques have been developed to identify S. moniliformis. The $16 \mathrm{~S}$ rRNA gene sequence has been used on a variety of specimens including heart valves, bone and synovial fluid, offering an alternative tool for rapid and efficient diagnosis. ${ }^{8,16,17}$ However, identification with this method is only specific to the Streptobacillus genus and not the species. ${ }^{17}$ As demonstrated in this case, PCR can help to identify S. moniliformis in the case of culture-negative mitral native valve endocarditis. Three other cases of both native and prosthetic valve $S$. moniliformis endocarditis were diagnosed using 16S rRNA PCR assays where the blood cultures remained negative after two weeks incubation. ${ }^{12-13,16}$

Although S. moniliformis PCR is not commercially available to be performed on blood samples, 16S rRNA sequencing for $S$. moniliformis from preliminary positive blood cultures has been suggested. ${ }^{3}$ In comparison to blood cultures, PCR can increase the sensitivity while decreasing the time to obtain results, which can take up to 7 days using standard techniques, although being significantly more expensive. ${ }^{18}$ Given the large sequence database of bacteria available from GenBank, the 16S rRNA is the favored target gene for the identification of fastidious organisms. Tak et al. compared

\section{References}

1. Budair B, Goswami K, Dhukaram V. Septic arthritis secondary to rat bite fever: a challenging diagnostic course. BMJ Case Rep 2014;2014.

2. Flannery D, Akinboyo I, Ty J, et al. Septic arthritis and concern for osteomyelitis in a child with rat bite fever. J Clin Microbiol 2013;51:1987-9.

3. Hayakawa Y, Suzuki J, Suzuki M, et al. A Case Study of Rat Bite Fever Caused by Streptobacillus moniliformis. Jpn J Infect Dis 2017;70:323-5.

4. Elliott S. Rat bite fever and Streptobacillus moniliformis. Clin Microbiol Rev 2007;20:13-22.

5. Wullenweber M. Streptobacillus moniliformis-a zoonotic pathogen. 
Taxonomic considerations, host species, diagnosis, therapy, geographical distribution. Lab Anim 1995;29:1-15.

6. Fenn D, Ramoutar A, Jacob G, Bin Xiao H. An unusual tale of rat-bite fever endocarditis. BMJ Case Rep 2014;2014.

7. Madhubashini M, George S, Chandrasekaran S. Streptobacillus moniliformis endocarditis: case report and review of literature. Indian Heart $\mathrm{J}$ 2013;65:442-6.

8. Boot R, Oosterhuis A, Thuis H. PCR for the detection of Streptobacillus moniliformis. Lab Anim 2002;36:200-8.

9. Rumley R, Patrone N, White L. Rat-bite fever as a cause of septic arthritis: a diagnostic dilemma. Ann Rheum Dis 1987;46:793-5.

10. Rupp M. Streptobacillus moniliformis endocarditis: case report and review. Clin Infect Dis 1992;14:769-72.

11. Rordorf T, Zuger C, Zbinden R, et al.
Streptobacillus moniliformis endocarditis in an HIV-positive patient. Infection 2000;28:393-4.

12. Chen P, Lee N, Yan J, et al. Prosthetic valve endocarditis caused by Streptobacillus moniliformis: a case of rat bite fever. $\mathrm{J}$ Clin Microbiol 2007;45:3125-6.

13. Ioffe V, Amir G, Zalzstein E, et al. Streptobacillus moniliformis Endocarditis: An Unusual Case of Pulmonary Valve Erosion Resulting in Free Pulmonary Regurgitation and Aneurysm. World J Pediatr Congenit Heart Surg 2016 [Epub ahead of print].

14. Abadeer M, Kouretas P, Woods R. Rat bite fever: Complex triple valve surgery for endocarditis and sinus of Valsalva fistula. J Thorac Cardiovasc Surg 2017;153:e45-e6.

15. Holroyd K, Reiner A, Dick J. Streptobacillus moniliformis polyarthritis mimicking rheumatoid arthritis: an urban case of rat bite fever. Am J Med 1988;85:711-4.

16. Kondruweit M, Weyand M, Mahmoud $\mathrm{F}$, et al. Fulminant endocarditis caused by Streptobacillus moniliformis in a young man. J Thorac Cardiovasc Surg 2007;134:1579-80.

17. Eisenberg T, Ewers C, Rau J, et al. Approved and novel strategies in diagnostics of rat bite fever and other Streptobacillus infections in humans and animals. Virulence 2016;7:630-48.

18. Faraji R, Behjati-Ardakani M, Moshtaghioun S, et al. The diagnosis of microorganism involved in infective endocarditis (IE) by polymerase chain reaction (PCR) and real-time PCR: A systematic review. Kaohsiung J Med Sci 2018;34:71-78.

19. Tak T, Shukla S. Molecular diagnosis of infective endocarditis: a helpful addition to the Duke criteria. J Clin Med Res 2004;2:206-8. 